ARTICLE

DOI: $10.1038 / \mathrm{s} 41467-018-03144-9$

OPEN

\title{
Induction of long-lived room temperature phosphorescence of carbon dots by water in hydrogen-bonded matrices
}

Qijun Li ${ }^{1}$, Ming Zhou ${ }^{1}$, Mingyang Yang ${ }^{1}$, Qingfeng Yang $^{1}$, Zhixun Zhang ${ }^{1} \&$ Jing Shi ${ }^{1}$

\begin{abstract}
Phosphorescence shows great potential for application in bioimaging and ion detection because of its long-lived luminescence and high signal-to-noise ratio, but establishing phosphorescence emission in aqueous environments remains a challenge. Herein, we present a general design strategy that effectively promotes phosphorescence by utilising water molecules to construct hydrogen-bonded networks between carbon dots (CDs) and cyanuric acid (CA). Interestingly, water molecules not only cause no phosphorescence quenching but also greatly enhance the phosphorescence emission. This enhancement behaviour can be explained by the fact that the highly ordered bound water on the CA particle surface can construct robust bridge-like hydrogen-bonded networks between the CDs and CA, which not only effectively rigidifies the $\mathrm{C}=\mathrm{O}$ bonds of the $\mathrm{CDs}$ but also greatly enhances the rigidity of the entire system. In addition, the CD-CA suspension exhibits a high phosphorescence lifetime $(687 \mathrm{~ms})$ and is successfully applied in ion detection based on its visible phosphorescence.
\end{abstract}

\footnotetext{
${ }^{1}$ State Key Laboratory of Tribology, School of Mechanical Engineering, Tsinghua University, Beijing 100084, China. Correspondence and requests for materials should be addressed to M.Z. (email: zhouming@tsinghua.edu.cn)
} 
$\mathrm{R}$ oom temperature phosphorescence (RTP) has attracted growing attention because of its long-lived luminescence, large stokes shift and high signal-to-noise ratio, allowing its application in the fields of optoelectronic devices ${ }^{1-4}$, bioimaging $^{5-7}$, chemical sensors ${ }^{8-10}$ and so forth. Most current RTP materials show phosphorescent features in their anhydrous solid states ${ }^{11-13}$, but phosphorescence quenching usually occurs in the presence of water owing to the presence of dissolved oxygen as well as the occurrence of solvent-assisted relaxation ${ }^{14}$, impeding the aqueous applications of RTP materials. Though few species with phosphorescence in aqueous media have been reported $^{15,16}$, most of them exhibit relatively short emission lifetimes. In addition, the present RTP materials are mainly organometallic ${ }^{4,17,18}$ and crystalline organic compounds ${ }^{19-21}$. However, all of these materials suffer from high costs, cytotoxicity and complicated preparation processes. Therefore, the development of a facile strategy for preparing efficient RTP materials in aqueous environments is desirable.

Recently, carbon dots (CDs) have aroused increasing attention as a new type of photoluminescent (PL) nanomaterial because of their high photostability, low toxicity and small size $22-24$. Generally, CDs show strong fluorescence, but minimal phosphorescence is observed at room temperature ${ }^{25-28}$. However, when $\mathrm{CDs}$ are fixed in a certain matrix, such as poly(vinyl alcohol $)^{29-32}, \mathrm{KAl}\left(\mathrm{SO}_{4}\right)_{2} \cdot \mathrm{x}\left(\mathrm{H}_{2} \mathrm{O}\right)^{33}$ or polyurethane $\mathrm{e}^{34}$, obvious phosphorescence can be detected under excitation by ultraviolet light. Most recently, in 2016, an ultralong phosphorescence lifetime (up to $1.06 \mathrm{~s}$ ) was achieved in our laboratory from CDs and composite matrices ${ }^{35}$. The matrix can effectively protect the CD triplet states from quenching by restricting their intermolecular motions. However, nearly all RTP materials based on CDs emit phosphorescence only in their dry state. Phosphorescence would undergo extensive quenching in the presence of water because water molecules usually break up hydrogen-bonding interactions between $\mathrm{CDs}$ and matrices. In addition, the dissolved oxygen present in water can further contribute to phosphorescence quenching. These unfavourable factors severely hinder the aqueous application of RTP materials, particularly in the fields of bioimaging and chemical sensors.

The water that exists in the vicinity of the matrix can generally be divided into three types: bulk water, freezing bound water, and non-freezing bound water. Bound water combines with proteins $^{36,37}$, phospholipids ${ }^{38}$ and other solid substances, ${ }^{39}$ mainly through hydrogen bonds. Compared with bulk water, bound water exhibits unique structural properties, including difficulty freezing, difficult separation, and restricted mobility and solubility, which endow it with unique applications ${ }^{36,40,41}$.
A recent study ${ }^{42}$ indicated that the bound water confined in a polyelectrolyte brush exhibited a tight arrangement with a high density, which greatly enhanced the hydrogen-bonded network. The strong hydrogen-bonding structures of water in swollen polymer brushes have been reported to suppress protein adsorption, affording biocompatibility ${ }^{43}$. Based on these conclusions, it is speculated that if we utilise bound water to construct hydrogen-bonded networks between CDs and matrices, effective phosphorescence may be achieved, even in aqueous environments. The achievement of such phosphorescence emission by utilising bound water is considered to be important in biological applications, such as cell imaging and disease diagnosis, as bound water widely exists in cells and biological tissues.

Herein, a facile strategy for preparing a long-afterglow RTP material by utilising water molecules to construct hydrogenbonded networks between CDs and cyanuric acid (CA) particles is reported. Our results show that water molecules not only cause no phosphorescence quenching but also greatly enhance the phosphorescence emission. This abnormal enhancement behaviour may be attributed to the water-induced formation of hydrogen-bonded networks between CDs and CA particles. In the presence of water, the surfaces of the CA particles can strongly absorb a layer of highly ordered water molecules (non-freezing bound water). These bound water molecules can construct robust bridge-like hydrogen-bonded networks between the CDs and CA particles, which not only effectively rigidifies the $\mathrm{C}=\mathrm{O}$ bonds of CDs but also greatly enhances the rigidity of the entire system, resulting in the enhancement of phosphorescence. In addition, the CD-CA suspension exhibits a phosphorescence lifetime of $687 \mathrm{~ms}$ under $373 \mathrm{~nm}$ excitation and is successfully applied in ion detection based on its visible phosphorescence. As an example, the detection limit of $\mathrm{Fe}^{3+}$ ions is as low as $32 \mu \mathrm{M}$. The CD-CA suspension can also be successfully applied to the determination of $\mathrm{Fe}^{3+}$ in real lake water and complicated protein solutions.

\section{Results}

Characterisation of the CDs and CD-CA powder. The preparation of CDs and the CD-CA powder is described in Fig. 1. The CD aqueous solution and CD-CA powder emitted blue light under illumination by UV (365 $\mathrm{nm}$ ) light. Interestingly, when the ultraviolet source was removed from the CD-CA powder, a green afterglow was observed with the naked eye at room temperature. The morphologies and optical properties of the CDs in solution and the CD-CA powder were studied. As shown in Fig. 2a, a transmission electron microscopy (TEM) image of the CDs demonstrates the formation of well-dispersed nanoparticles with

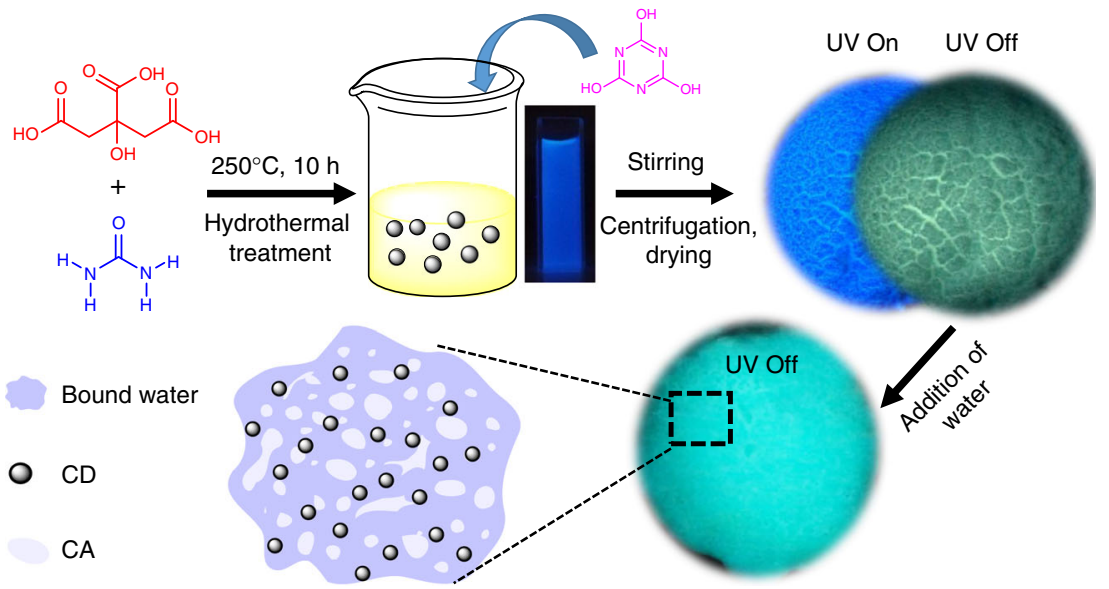

Fig. 1 Preparation of the CDs and the CD-CA system. Schematic illustration for the preparation of the CDs and the CD-CA system 
a

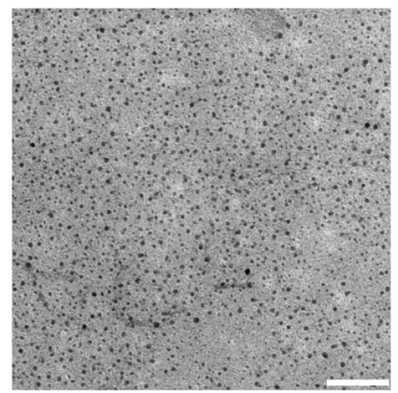

d

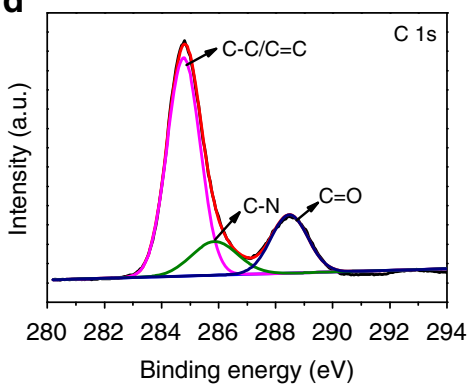

b

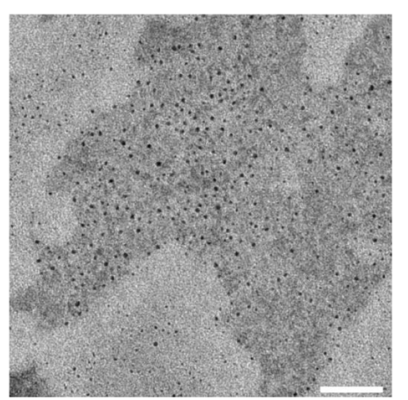

e

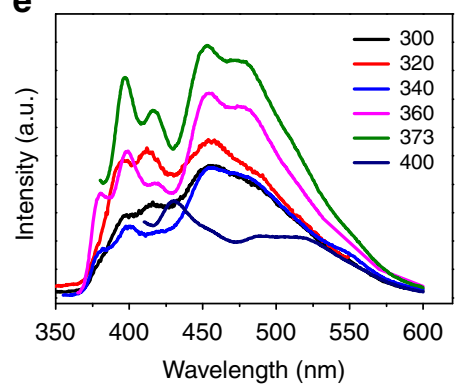

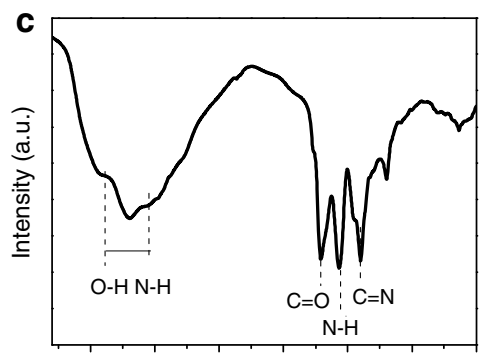

350030002500200015001000500

Wavenumber $\left(\mathrm{cm}^{-1}\right)$

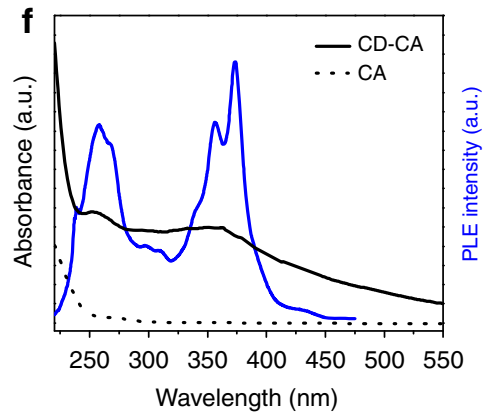

Fig. 2 Characterisation of the CDs and CD-CA powder. a TEM image of the CDs (scale bar, $50 \mathrm{~nm}$ ). $\mathbf{b}$ TEM image of the CD-CA nanohybrid (scale bar, 50 $\mathrm{nm}$ ). c FTIR spectrum of the CDs. d High-resolution C $1 \mathrm{~s}$ XPS spectrum of the CDs. e Phosphorescence spectra of the CD-CA powder under different excitation wavelengths. f Phosphorescence excitation spectrum (blue solid line) of the CD-CA powder with emission at $480 \mathrm{~nm}$ and the absorption spectra of the CD-CA power (black solid line) and the CA powder (black dotted line)

a mean particle diameter of $4.8 \mathrm{~nm}$ (Supplementary Fig. 1a). An atomic force microscopy image of the CDs reveals that their topographic heights are in the range of $0.5-2.5 \mathrm{~nm}$, suggesting that the prepared CDs are anisotropic (Supplementary Fig. 1b). The related high-resolution TEM (HRTEM) image and X-ray diffraction (XRD) results reveal the presence of both crystalline graphite and amorphous phases (Supplementary Fig. 1c, d). In the presence of CA, the CDs evidently cover the surface of CA in their assembled state, and the morphology of the CDs mediated by CA exhibits no significant changes relative to that of the original CDs (Fig. 2b). The Fourier transform infrared (FTIR) spectrum of the CDs (Fig. 2c) displays characteristic peaks at 1705,1565 and $1400 \mathrm{~cm}^{-1}$ that are attributed to $\mathrm{C}=\mathrm{O}, \mathrm{N}-\mathrm{H}$, and $\mathrm{C}-\mathrm{N}$ stretching vibrations, respectively. The results of $\mathrm{X}$-ray photoelectron spectroscopy (XPS) experiments indicate that the CDs mainly consist of carbon, nitrogen, and oxygen (Supplementary Fig. 1e). The C $1 \mathrm{~s}$ XPS spectrum (Fig. 2d) shows three peaks at $284.6,285.6$ and $288.1 \mathrm{eV}$ for $\mathrm{C}-\mathrm{C}, \mathrm{C}-\mathrm{N}$ and $\mathrm{C}=\mathrm{O}$, respectively ${ }^{44}$. Fig $2 \mathrm{e}$ shows the corresponding phosphorescence spectra of the CD-CA powder under different excitation wavelengths, where an optimal excitation at $373 \mathrm{~nm}$ was obtained. To investigate the origin of the observed phosphorescence, the phosphorescence excitation and absorption spectra of the CD-CA powder were investigated (Fig. 2f). In the absorption spectrum, two bands centred at 255 and $360 \mathrm{~nm}$ were observed, which are owing to the $\pi-\pi^{*}$ transition of the aromatic $s p^{2}$ domains and the $n-\pi^{*}$ transition of the $\mathrm{C}=\mathrm{O}$ bonds, respectively ${ }^{45}$. The phosphorescence emission of the CD-CA powder at $480 \mathrm{~nm}$ could be excited within the range of $300-400 \mathrm{~nm}$, which overlaps with the absorption bands of $\mathrm{C}=\mathrm{O}$, suggesting that the observed phosphorescence may come from the surface $\mathrm{C}=\mathrm{O}$ bonds of the $\mathrm{CDs}$.

The role of water in phosphorescence. The phosphorescence of the CD-CA powder could be significantly enhanced by adding a certain amount of water. Notably, the intensity of phosphorescence in the presence of water was nearly three times higher than that of the dry $\mathrm{CD}-\mathrm{CA}$ powder, and the average lifetime increased from $0.253 \mathrm{~s}$ for the dry CD-CA powder to $0.687 \mathrm{~s}$ for the water-added CD-CA powder (Fig. 3a, b), whereas this enhancement was not observed with other common organic solvents (e.g., ethanol, methanol, acetone, and tetrahydrofuran) (Supplementary Fig. 2a). This interesting water-triggered phosphorescence feature endows the CDs with great potential for application in optical recording media and double anticounterfeiting (Supplementary Fig. $2 \mathrm{~b}$ and Supplementary Note 1). Water molecules are known to usually break up intermolecular hydrogen-bonding interactions, resulting in a significant decrease in phosphorescence. However, the distinctive enhancement property observed here may be attributed to the formation of unique intermolecular interactions among the CDs, CA particles and water molecules. First, the possibility that the phosphorescence enhancement was caused by a change in the concentration before and after the addition of water was ruled out because the absorption spectra of the material in the absence and presence of water are almost identical (Supplementary Fig. 2c). Moreover, nearly identical PL decay curves were obtained before and after the addition of water, further excluding the selfquenching of the CDs in the dry state (Supplementary Fig. 2c). In addition, the zeta potential results indicate that both the CDs and CA particles have negative potentials in aqueous solution (Supplementary Table 1), indicating the presence of repulsive electrostatic forces between the CDs and CA particles; thus, the possibility that the phosphorescence enhancement in this experiment was induced by electrostatic attraction is also unlikely. When we tried to enhance the phosphorescence of the CD-CA powder by adding plenty of water, we found that the phosphorescence enhancement was not linearly related to the water content. As shown in Fig. 3a, an obvious enhancement in the phosphorescence was observed for a $20 \%$ water content, and the intensity of the phosphorescence remained nearly unchanged with a further increase in the water content up to $70 \%$, but when 

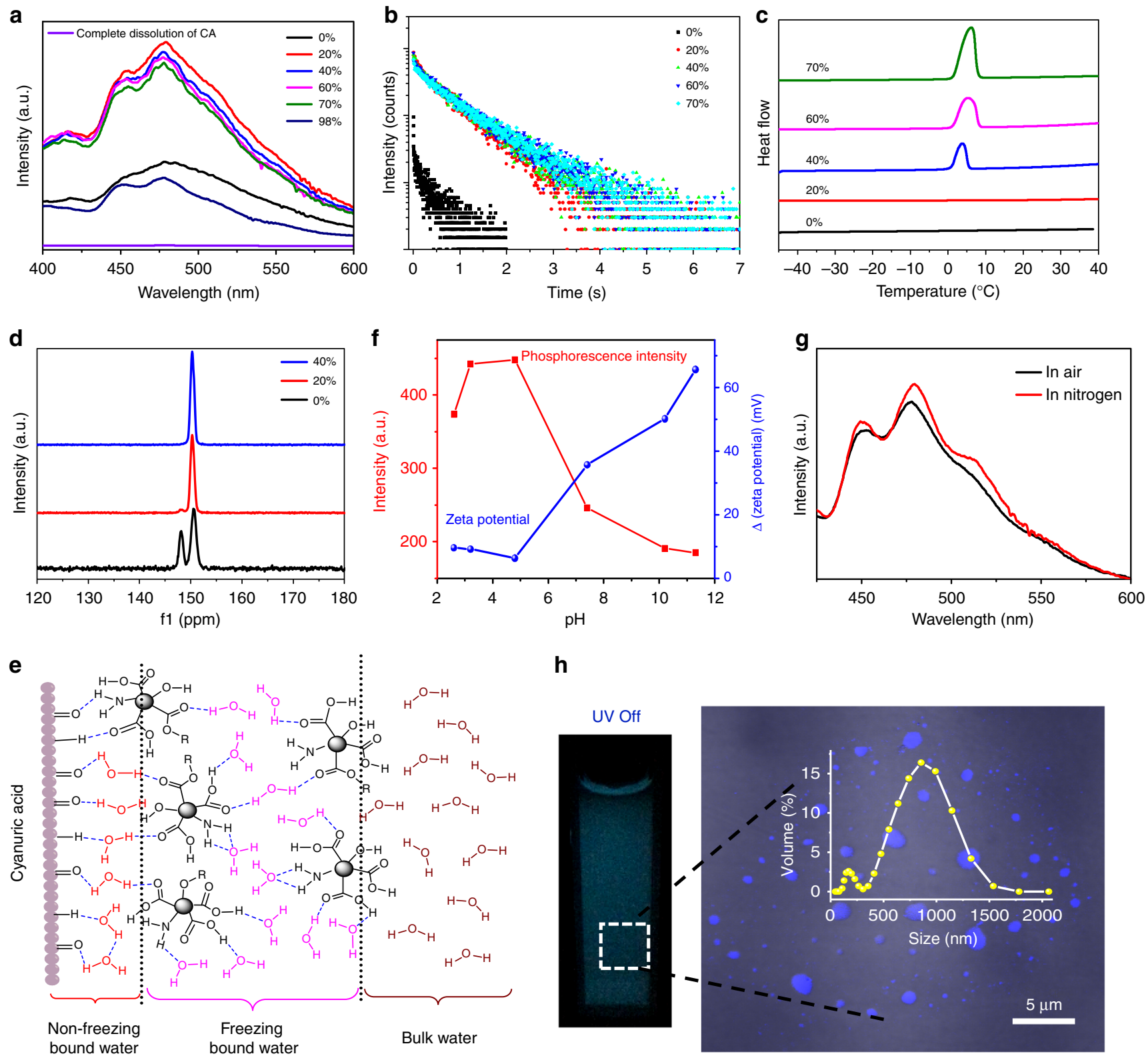

h

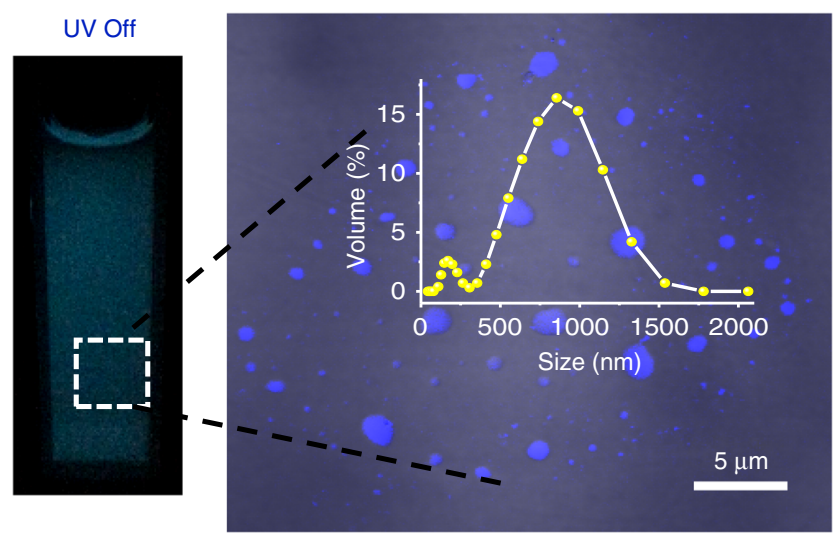

Fig. 3 Role of water in phosphorescence. a Phosphorescence spectra and $\mathbf{b}$ lifetime decay profiles of the CD-CA system with various water contents under excitation at $373 \mathrm{~nm}$. c Representative DSC-heating curves of the CD-CA system with various water contents as a function of temperature. $\mathbf{d}{ }^{13} \mathrm{C}$ NMR spectra of the CD-CA system with various water contents. e Schematic illustration of the molecular interactions between the CDs, CA particles and water molecules. $\mathbf{f}$ Phosphorescence intensity at $480 \mathrm{~nm}$ of the $\mathrm{CD}-\mathrm{CA}$ suspension as a function of the $\mathrm{pH}$ (red line) and the sum of the absolute values of the $\mathrm{CD}$ and $\mathrm{CA}$ zeta potentials as a function of $\mathrm{pH}$ (blue line). $\mathbf{g}$ Phosphorescence spectra of the CD-CA suspension in air-saturated and nitrogen ( $\left.\mathrm{N}_{2}\right)$ conditions under excitation at $373 \mathrm{~nm}$. h Image (left) of the CD-CA suspension treated by ultrasound and low-speed centrifugation after the UV light was turned off; corresponding fluorescence image (right), as determined by CLSM; corresponding size distribution (inset), as determined by DLS

the CD-CA powder dissolved after adding a large amount of water $(98 \%)$, the phosphorescence decreased and disappeared completely in the dissolved state of the CD-CA powder. To explain the mechanism, we investigated the different states of water present in the $\mathrm{CD}-\mathrm{CA}$ system by differential scanning calorimetry (DSC) (Fig. 3c). An endothermic melting peak was not observed at a low water content $(20 \%)$, suggesting that this portion of water is of the non-freezing bound type. As the content of water gradually increased from $20 \%$ to $70 \%$, a broad endothermic peak appeared and moved to higher temperatures, which indicates the presence of two states of freezing water (freezing bound water and bulk water) in the composite. More robust exothermic signals were observed at high water contents, corresponding to an increase in the bulk water content. Although the quantification of each state of water by DSC is difficult due to the overlap between the two melting peaks, we can confirm that all the water in the sample with a $20 \%$ water content is nonfreezing bound water. Based on the results presented above, the observed phosphorescence enhancement is mainly attributed to non-freezing bound water, while bulk water had a negligible effect on the phosphorescence. A possible mechanism for the waterenhanced phosphorescence emission is illustrated in Fig. 3e. CA is a unique cyclic amide consisting of three hydrogen bond donors and three acceptor sites, leading to a range of hydrogenbonding possibilities. In the presence of water, the CA particle surfaces can strongly absorb a layer of highly ordered water 
molecules (non-freezing bound water). This type of water exhibits unique structural properties, including difficulty freezing, difficult separation, and restricted mobility and solubility, and constructs robust bridge-like hydrogen-bonded networks between the CDs and CA particles, which not only effectively rigidifies the $\mathrm{C}=\mathrm{O}$ bonds of CDs but also greatly enhances the rigidity of the entire system, resulting in the enhancement of phosphorescence. To further demonstrate the bridging role of the water molecules, ${ }^{13} \mathrm{C}$ NMR spectra of the CD-CA system were measured (Fig. 3d). In the dry state, the ${ }^{13} \mathrm{C}-\mathrm{NMR}$ spectrum of CD-CA contains two distinct, non-equivalent peaks at 148.1 and $150.6 \mathrm{ppm}$ that arise from the different hydrogen-bonding environments in the supramolecular assembly (the hydrogen bonds formed by CA with itself through different sites and those formed between CA and CDs). With an increase in the water content from 0 to $40 \%$, the peak at $148.1 \mathrm{ppm}$ becomes weaker and shifts to $150.2 \mathrm{ppm}$. Such a phenomenon can be explained by the fact that water molecules can construct robust bridge-like hydrogen-bonded networks between the CDs and CA particles and replace the hydrogen bonds formed by CA with itself or between CA and CDs, resulting in the disappearance of the asymmetric hydrogenbonding interactions that are present in the CD-CA dry state. In addition, the Raman spectra of CA further demonstrated the presence of hydrogen-bonding interactions between water molecules and the CA particles (Supplementary Fig. 2d and Supplementary Note 1). Compared with bound water, bulk water is not intimately bound to the CA particle surface but rather interacts weakly with the CA particles, leading to a negligible effect on phosphorescence. However, when dissolution of the CD-CA powder occurred, the number of water molecules bound to the CA particles began to decrease until they were all converted to bulk water in the completely dissolved state. At this point, the hydrogen-bonded network between the CA particles and CDs collapsed, resulting in the disappearance of phosphorescence.

To obtain better insight into the bonding interactions between the CDs and CA molecules in the completely dissolved state of the CD-CA powder, their thermodynamic parameters and binding constants were measured by isothermal titration calorimetry (ITC) and microscale thermophoresis (MST) (Supplementary Fig. 3a-d and Supplementary Note 1). The obtained endothermic signal and positive entropy values suggest that the binding of CDs to CA molecules is partly driven by hydrophobic interactions, which may be attributed to the hydrophobic carbon skeleton of the CDs. A low binding constant indicates the presence of weak binding interactions between the CDs and CA, explaining the absence of phosphorescence in the completely dissolved state of the CD-CA powder.

The optical spectra of CD-CA powders with various $C D$ contents were recorded (Supplementary Fig. $4 \mathrm{a}$ and Table 2 and Supplementary Note 1). At low CD concentrations, the phosphorescence lifetime and fluorescence quantum yield remained nearly unchanged, whereas both properties showed obvious quenching at high concentrations, which is owing to an increase in the level of self-absorption by the CDs. In addition, we investigated the effects of the aqueous environment $\mathrm{pH}$ on phosphorescence. The emission intensity of the CD-CA system showed a negligible change over the $\mathrm{pH}$ range from 3.1 to 4.8 and a sudden reduction at $\mathrm{pH}$ 7.4. To explain this phenomenon, the zeta potentials of the CDs and CA particles were measured. The sum of the absolute values of the $\mathrm{CD}$ and CA particle zeta potentials exhibited an opposite trend as that of the emission intensities of the CD-CA system at different $\mathrm{pH}$ values (Fig. $3 \mathrm{f}$ and Supplementary Table 1), suggesting that electrostatic interactions have an important role in phosphorescence. In addition, an obvious reduction in the amount of bound water in the CD-CA system at $\mathrm{pH} 7.4$ compared with that at $\mathrm{pH} 4.8$, which causes a further reduction in the phosphorescence of the CD-CA system at $\mathrm{pH}$ 7.4, was detected by DSC tests (Supplementary Fig. 4b). Dissolved oxygen is known to often cause phosphorescence quenching in water. Thus, the phosphorescence spectra of CD-CA suspensions with and without nitrogen bubbling were measured, and almost identical optical curves were obtained (Fig. $3 \mathrm{~g}$ ), which may be attributed to the restricted solubility of oxygen in bound water. In a further set of experiments, we investigated the effects of the temperature on the photoluminescence of the CD-CA system containing $40 \%$ water in the range of 78-301 K. (Supplementary Fig. 4c, d, Table 3 and Supplementary Note 1). Usually, the phosphorescence intensity increases as the temperature decreases ${ }^{30,46}$; however, our results were different. As shown in Supplementary Fig. 3c, the phosphorescence of the $\mathrm{CD}-\mathrm{CA}$ system decreased with a decrease in the temperature, which might be attributed to the freezing water in the CD-CA system. Small phosphorescent materials are very important in biology and related fields. The phosphorescence image, confocal laser scanning microscopy (CLSM) results and dynamic light scattering (DLS) data (Fig. 3h) illustrated that the CD-CA suspension still exhibited visible phosphorescence under illumination by UV light at the nanometre scale, which is crucial for real applications.

Application in ion detection. Ion detection based on CD fluorescence has recently undergone rapid development ${ }^{23,47}$, but this application can be conducted in only non-fluorescent aqueous solutions ${ }^{48,49}$. Many proteins in living organisms and algae in polluted water have strong fluorescence emissions, leading to the failure of the fluorescent probe. Phosphorescent detection avoids interference from the short-lifetime fluorescent background or scattered light in real samples ${ }^{50}$, providing a higher sensitivity and signal-to-noise ratio of detection in complicated media. Taking advantage of the long-lived persistent RTP in aqueous media, the CD-CA suspension could be utilised as a sensor for the detection of metal ions in water. The test results indicate that the phosphorescence of the CD-CA suspension can be selectively quenched in the presence of $\mathrm{Fe}^{3+}$ (Fig. 4a). The phosphorescence intensity gradually decreased as the concentration of $\mathrm{Fe}^{3+}$ ions increased (Fig. 4b). A good linear relationship within the range from 0.1 to $0.8 \mathrm{mM}$ with a correlation coefficient $\left(R^{2}\right)$ of 0.9891 was obtained (Fig. 4c). The limit of detection was estimated to be $32 \mu \mathrm{M}$ based on the rule of three times the standard deviation. We also investigated the phosphorescence quenching effect of other metal ions on the CD-CA suspension. As shown in Fig. $4 \mathrm{a}, \mathrm{Fe}^{3+}$ ions had the strongest ability to quench the phosphorescence, while other ions showed a negligible quenching effect. In addition, we found that the fluorescence of the CDs could also be selectively be quenched by $\mathrm{Fe}^{3+}$ (Fig. $4 \mathrm{~d}$, e). The simultaneous fluorescence and phosphorescence quenching by $\mathrm{Fe}^{3+}$ was not a coincidence. The quenching effect presumably results from static quenching arising from the formation of a non-fluorescent complex between the $\mathrm{CDs}$ and $\mathrm{Fe}^{3+}$. This non-luminescent chelate changes the original CD energy level structure created by the surface carbonyl or hydroxyl groups. Combining the above analysis, the $\mathrm{C}=\mathrm{O}$ bonds on the $\mathrm{CD}$ surface are mainly responsible for the observed phosphorescence. Thus, the change in the energy level structure leads to phosphorescence quenching. To gain further insight into the PL quenching mechanism, the fluorescence lifetime, phosphorescence lifetime and UV-vis absorption spectra were obtained (Supplementary Fig. 5a-c). The fluorescence lifetime of the CDs remained nearly constant before and after the coordination of $\mathrm{Fe}^{3+}$ ions, as did the phosphorescence lifetime of the CD-CA suspension. Moreover, the absorption peaks of the CDs showed obvious differences in the 

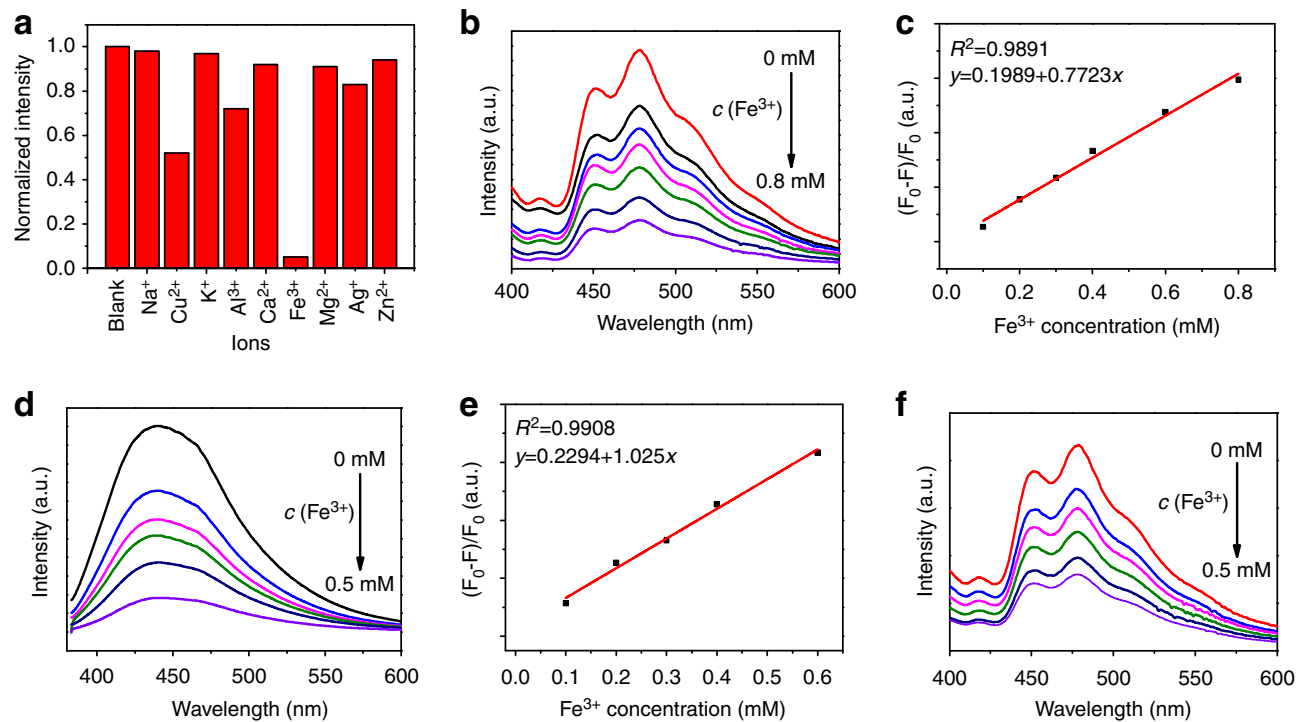

Fig. 4 Detection of $\mathrm{Fe}^{3+}$ ions. a Normalised phosphorescence intensity of the CD-CA suspension in the presence of various metal ions. $\mathbf{b}$ Phosphorescence spectra of CD-CA suspensions with various calculated concentrations of $\mathrm{Fe}^{3+}$; from top to bottom: 0, 0.1, 0.2, 0.3, 0.4, 0.5 and 0.8 mM. c Stern-Volmer plot as a function of $\mathrm{Fe}^{3+}$ concentration. $\mathbf{d}$ Fluorescence spectra of $\mathrm{CDs}$ at various calculated concentrations of Fe ${ }^{3+}$; from top to bottom: $0,0.1,0.2,0.3$, 0.4 and $0.5 \mathrm{mM}$. e Stern-Volmer plot as a function of $\mathrm{Fe}^{3+}$ concentration. $\mathbf{f}$ Phosphorescence spectra of CD-CA suspensions at various calculated concentrations of $\mathrm{Fe}^{3+}$ in a peptone solution; from top to bottom: $0,0.1,0.2,0.3,0.4$ and $0.5 \mathrm{mM}$

absence and presence of $\mathrm{Fe}^{3+}$. These results clearly indicate that the quenching mechanism of the CDs by $\mathrm{Fe}^{3+}$ occurs through a static quenching process.

In further experiments, we applied the CD-CA probe to $\mathrm{Fe}^{3+}$ determination in complicated peptone solutions containing numerous amino acids and proteins. As anticipated, the phosphorescent sensor still showed a good linear correlation in the concentration range from $0.1-0.5 \mathrm{mM}$ with a detection limit of $28 \mu \mathrm{M}$, but the fluorescent sensor based on CDs did not work well because of interference by scattering light and fluorescent organics (Fig. $4 \mathrm{f}$ and Supplementary Fig. 5d, e). In addition, the CD-CA suspension was also successfully applied to the determination of $\mathrm{Fe}^{3+}$ in real lake water (Supplementary Fig. 5f).

Universality confirmation. To confirm the universality of our technology, we designed a series of phosphorescent materials containing a variety of host-guest materials. The materials a$\mathrm{CD}^{31}, \mathrm{~b}-\mathrm{CD}^{35}$ and c-CD $\mathrm{CD}^{32}$ have been reported in previous work, and a full description of their syntheses is described in the Supplementary Information. As anticipated, the prepared materials all exhibited long phosphorescence lifetimes in solution (Fig. 5a, b, Supplementary Table 4 and Supplementary Note 1), indicating that our method can be generally applied to the establishment of RTP in metal-free organic materials in aqueous environments.

\section{Discussion}

In summary, we have developed an efficient metal-free RTP material system in aqueous media by introducing multiple rationally designed hydrogen-bonding interactions between the $\mathrm{CDs}, \mathrm{CA}$ and water molecules. The CA particle surfaces contain a large number of polar groups; thus, CA particles adsorb a layer of highly ordered water molecules (non-freezing bound water) through strong hydrogen-bonding interactions. In the presence of $\mathrm{CDs}$, the particles can form robust hydrogen-bonded networks, which not only effectively rigidifies the $\mathrm{C}=\mathrm{O}$ bonds of the $\mathrm{CDs}$ but also greatly enhances the rigidity of the entire system, leading to an enhancement of the phosphorescence. The CD-CA suspension exhibited a high phosphorescence lifetime of $687 \mathrm{~ms}$
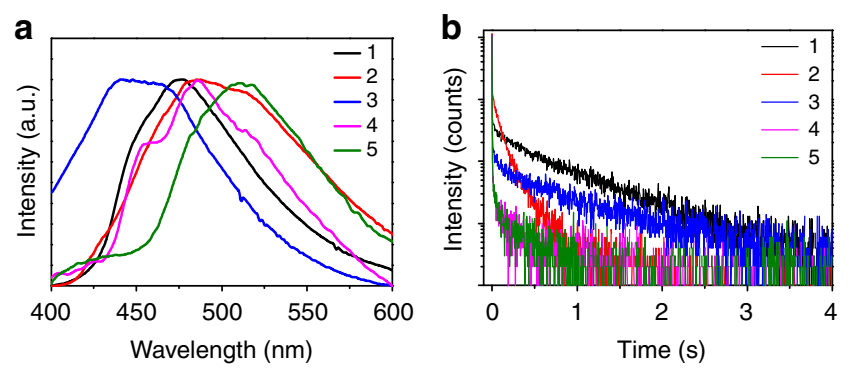

Fig. 5 Photoluminescence properties of a series of materials in an aqueous environment. a Normalised phosphorescence intensity of a series of phosphorescent materials in an aqueous environment. b Lifetime decay profiles of a series of phosphorescent materials in an aqueous environment

under $373 \mathrm{~nm}$ excitation and was successfully applied in ion detection based on its visible phosphorescence. Phosphorescence detection more effectively eliminates the fluorescence background and scattered light than the fluorescence method. Therefore, our $\mathrm{CD}-\mathrm{CA}$ system shows great potential for the detection of $\mathrm{Fe}^{3+}$ in real lake water and complicated protein solutions. We believe that the demonstrated strategy based on bound water for enabling effective RTP in aqueous media can be utilised for the development of novel metal-free organic phosphorescence materials for chemical sensors as well as for bioimaging applications in real water environments.

\section{Methods}

Chemicals. Unless otherwise noted, all chemicals were commercially available and used as received without additional purification. Anhydrous citric acid, urea, CA, folic acid, ethylenediamine triacetic acid (EDTA)-2Na, biuret and the oxidised form of $\beta$-nicotinamide-adenine dinucleotide were purchased from J\&K Scientific Ltd (Beijing) or Aladdin Chemicals Ltd (Shanghai). Deionised (DI) water (18.2 $\mathrm{M} \Omega . \mathrm{cm}$ at $25^{\circ} \mathrm{C}$ ) prepared by a Milli-Q water system was used throughout all experiments. Lake water samples were obtained from the Weiminghu Lake of Peking University, China. Before evaluation, the lake water samples were filtered through a $0.20 \mu \mathrm{m}$ membrane and then centrifuged at $10,000 \mathrm{rpm}$ for $10 \mathrm{~min}$. The resultant water samples were spiked with $\mathrm{Fe}^{3+}$ at different concentrations and then analysed with the proposed method. 
Material characterisation. TEM images were taken by an H-7650 instrument (Hitachi, Japan). HRTEM images were obtained on a JEM2010 high-resolution field-emission transmission electron microscope at $200 \mathrm{KV}$ (JEOL, Japan). XPS was performed using an Escalab 250 XI system (Thermo Electron Corporation, USA). FTIR spectra were recorded on a Bruker Tensor 27 spectrophotometer (Germany). The absorbance spectra were recorded with a Shimadzu UV-3600 spectrometer. Steady-state PL and phosphorescence spectra were collected on a Hitachi F-7000 fluorescence spectrophotometer. Time-resolved luminescence spectra were obtained using an Edinburgh FLS920 fluorescence spectrophotometer. Lifetime decay profiles were measured using an Edinburgh FLS920 fluorescence spectrophotometer equipped with a xenon arc lamp (Xe900), a nanosecond hydrogen flash-lamp (nF920) and a microsecond flash-lamp ( $\mu$ F900). For the temperature dependent experiment, the sample was placed in an Optistat DN-V liquid nitrogen cryostat with a temperature control range between 80 and $293 \mathrm{~K}$. The lifetimes $(\tau)$ of the luminescence were obtained by fitting the decay curve with the following multi-exponential decay function:

$$
I(t)=\sum_{i} A_{i} \mathrm{e}^{-\frac{\mathrm{t}}{\tau_{\mathrm{i}}}}
$$

where $A_{i}$ and $\tau_{i}$ represent the amplitude and lifetime, respectively, of the individual components of the multi-exponential decay profiles. DSC was performed on a DSC Q-2000 from TA Instruments using cooling and heating rates of $5 \mathrm{~K} \mathrm{~min}^{-1}$. DLS was performed on a Malvern Instruments Zeta Sizer Nano-ZS apparatus. ITC was performed on the Micro Cal ITC-200. Fluorescence images were taken by a CLSM equipped with a diode laser at $405 \mathrm{~nm}$. The binding affinity study was investigated with an MST instrument. Raman spectra were measured on a Horiba-HR-800 Raman spectroscopy system. This system was equipped with a $514 \mathrm{~nm}$ diode laser source. NMR spectra were collected with a Bruker Avance $\amalg$ HD $500 \mathrm{MHz}$ instrument. XRD patterns were obtained by a Rigaku S2 diffractometer.

Synthesis of CDs. Anhydrous citric acid ( $1 \mathrm{~g})$ and urea $(0.5 \mathrm{~g})$ were dissolved in DI water $(25 \mathrm{~mL})$ and stirred to form a clear solution. Then, the solution was transferred to a poly (tetrafluoroethylene) (Teflon)-lined autoclave $(50 \mathrm{~mL}$ ) and heated at $250{ }^{\circ} \mathrm{C}$ for $10 \mathrm{~h}$. The obtained dark brown solution was centrifuged at high speed $\left(10,000 \mathrm{rad} \mathrm{min}^{-1}\right)$ for $20 \mathrm{~min}$ to remove large or agglomerated particles. The final products were obtained via freeze-drying. A stock solution $\left(4 \mathrm{mg} \mathrm{ml}^{-1}\right)$ of the obtained CDs was then prepared for further use.

Preparation of CD-CA powders. Different masses of the CDs $(0.2 \mathrm{mg}, 0.4 \mathrm{mg}, 0.8$ $\mathrm{mg}, 2 \mathrm{mg}, 4 \mathrm{mg}$, and $16 \mathrm{mg}$ ) were first diluted with $4 \mathrm{~mL}$ of DI water and mixed with CA $(0.75 \mathrm{~g})$ under constant stirring for $12 \mathrm{~h}$. The reaction mixtures were then centrifuged $(8000 \mathrm{rpm}, 10 \mathrm{~min})$ to remove the unabsorbed CDs, and the solid powders remaining on the bottoms of the centrifuge tubes were fully dried under vacuum. Finally, the CD-CA powders were obtained and then redissolved in water for further use. Unless stated otherwise, the CD-CA powder in this article was prepared by mixing CDs $(2 \mathrm{mg})$ and CA $(0.75 \mathrm{~g})$ in $4 \mathrm{~mL}$ of DI water.

Synthesis of a-CDs. The a-CDs were prepared according to previous work. EDTA-2Na $(1.6 \mathrm{~g})$ was placed in a quartz boat and heated in a tube furnace under a $\mathrm{N}_{2}$ atmosphere at a rate of $10^{\circ} \mathrm{C} \mathrm{min}{ }^{-1}$ to $400{ }^{\circ} \mathrm{C}$. After annealing for $4 \mathrm{~h}$ at $400{ }^{\circ} \mathrm{C}$, the reaction was allowed to cool to room temperature, and the black product was dispersed in DI water $(80 \mathrm{~mL})$ under ultrasonication for $30 \mathrm{~min}$. Then, the solution was centrifuged at $9500 \mathrm{rpm}$ for $16 \mathrm{~min}$ to remove larger and insoluble particles. Solid-state luminescent CDs were obtained via freeze-drying.

Synthesis of b-CDs. The b-CDs were prepared according to previous work. In brief, folic acid (FA) $(1 \mathrm{~g})$ was dissolved in DI water $(100 \mathrm{~mL})$. Then, the solution was transferred to a Teflon-lined autoclave $(200 \mathrm{~mL})$ and heated at $260{ }^{\circ} \mathrm{C}$ for $2 \mathrm{~h}$. After the reaction, the reactor was cooled to room temperature naturally. The final product was collected following centrifugation at $10,000 \mathrm{rpm}$ for $20 \mathrm{~min}$.

Synthesis of c-CDs. The c-CDs were prepared according to previous work. Isophorone diisocyanate $(8 \mathrm{~g}, 36 \mathrm{mmol})$ was added to a Teflon vessel that was sealed with an explosion-proof enclosure. Then, raw $\mathrm{N}$-doped carbon quantum dots (CQDs) were prepared under $700 \mathrm{~W}$ microwave irradiation at $250{ }^{\circ} \mathrm{C}$ for $10 \mathrm{~min}$. The oil-soluble CQDs were collected after centrifugation and dialysed. Then, the oil-soluble CQDs were ultrasonically dispersed in DI water $(50 \mathrm{~mL})$ and heated at $180^{\circ} \mathrm{C}$ for $3 \mathrm{~h}$. After the reaction, the mixture was cooled to room temperature, and the obtained yellow solution was centrifuged $(10,000 \mathrm{rpm}, 20 \mathrm{~min})$ and filtered through a $0.22 \mathrm{~mm}$ filter membrane to remove large and insoluble particles. Solidstate luminescent a-CDs were obtained via freeze-drying.

Fluorescent detection of $\mathbf{F e}^{\mathbf{3}+}$ ions. The detection of $\mathrm{Fe}^{3+}$ was performed in an ultrapure water solution. An aqueous solution of CDs $\left(0.01 \mathrm{mg} \mathrm{mL}^{-1}\right)$ was used as the blank solution. Different concentrations of $\mathrm{Fe}^{3+}$ were added to the CD blank solution, and the mixed solutions were equilibrated for $10 \mathrm{~min}$. In addition, the PL emission spectra were collected at an excitation wavelength of $373 \mathrm{~nm}$. The selectivity for $\mathrm{Fe}^{3+}$ was confirmed by adding other metal ion stock solutions instead of $\mathrm{Fe}^{3+}$ in a similar way. All experiments were performed at room temperature.

Phosphorescent detection of $\mathbf{F e}^{\mathbf{3}+}$ ions. The detection of $\mathrm{Fe}^{3+}$ was performed in an ultrapure water solution. Different concentrations of $\mathrm{Fe}^{3+}$ were added to $3 \mathrm{~mL}$ of the $\mathrm{CD}$ solution $\left(0.01 \mathrm{mg} \mathrm{mL}^{-1}\right)$, and the mixed solutions were equilibrated for 10 min. Then, the same amount of CA powder $(0.3 \mathrm{~g})$ was added to each mixed solution, which was stirred for $1 \mathrm{~h}$. Then, the PL emission spectra were collected at an excitation wavelength of $373 \mathrm{~nm}$. The selectivity for $\mathrm{Fe}^{3+}$ was confirmed by adding other metal ion stock solutions instead of $\mathrm{Fe}^{3+}$ in a similar way. All experiments were performed at room temperature. The detection of $\mathrm{Fe}^{3+}$ in lake water and complicated protein solutions was carried out using lake water and complicated protein solutions, respectively, instead of ultrapure water. The complicated protein solution was prepared by adding peptone $(0.5 \mathrm{~g})$ to ultrapure water $(50 \mathrm{~mL})$.

Data availability. The data that support the findings of this study are available from the corresponding author.

Received: 28 February 2017 Accepted: 23 January 2018

Published online: 21 February 2018

\section{References}

1. Baldo, M. A. et al. Highly efficient phosphorescent emission from organic electroluminescent devices. Nature 395, 151-154 (1998).

2. Wei, J. et al. Induction of strong long-lived room-temperature phosphorescence of N-phenyl-2-naphthylamine molecules by confinement in a crystalline Dibromobiphenyl matrix. Angew. Chem. Int. Ed. Engl. 55, 15589-15593 (2016)

3. Kabe, R., Notsuka, N., Yoshida, K. \& Adachi, C. Afterglow organic lightemitting diode. Adv. Mater. 28, 655-660 (2016).

4. $\mathrm{Xu}, \mathrm{H}$. et al. Recent progress in metal-organic complexes for optoelectronic applications. Chem. Soc. Rev. 43, 3259-3302 (2014).

5. Zhang, G., Palmer, G. M., Dewhirst, M. W. \& Fraser, C. L. A dual-emissive materials design concept enables tumour hypoxia imaging. Nat. Mater. 8, 747-751 (2009).

6. Xiong, X. et al. Thermally activated delayed fluorescence of fluorescein derivative for time-resolved and confocal fluorescence imaging. J. Am. Chem. Soc. 136, 9590-9597 (2014).

7. Palner, M., Pu, K., Shao, S. \& Rao, J. Semiconducting polymer nanoparticles with persistent near-infrared luminescence for in vivo optical imaging. Angew. Chem. Int. Ed. 127, 11639-11642 (2015).

8. Zhang, G. et al. Multi-emissive difluoroboron dibenzoylmethane polylactide exhibiting intense fluorescence and oxygen-sensitive room-temperature phosphorescence. J. Am. Chem. Soc. 129, 8942-8943 (2007).

9. Shu, X., Chen, Y., Yuan, H., Gao, S. \& Xiao, D. H2O2 sensor based on the room-temperature phosphorescence of Nano $\mathrm{TiO} 2 / \mathrm{SiO} 2$ composite. Anal. Chem. 79, 3695-3702 (2007).

10. Yang, X. \& Yan, D. Long-afterglow metal-organic frameworks: reversible guest-induced phosphorescence tunability. Chem. Sci. 7, 4519-4526 (2016).

11. Lee, D. et al. Room temperature phosphorescence of metal-free organic materials in amorphous polymer matrices. J. Am. Chem. Soc. 135, 6325-6329 (2013).

12. Kwon, M. S. et al. Suppressing molecular motions for enhanced roomtemperature phosphorescence of metal-free organic materials. Nat. Commun 6, 8947 (2015).

13. Kwon, M. S., Lee, D., Seo, S., Jung, J. \& Kim, J. Tailoring intermolecular interactions for efficient room-temperature phosphorescence from purely organic materials in amorphous polymer matrices. Angew. Chem. Int. Ed. Engl. 53, 11177-11181 (2014).

14. Mukherjee, S. \& Thilagar, P. Recent advances in purely organic phosphorescent materials. Chem. Commun. 51, 10988-11003 (2015).

15. Pfister, A., Zhang, G., Zareno, J., Horwitz, A. F. \& Fraser, C. L. Boron polylactide nanoparticles exhibiting fluorescence and phosphorescence in aqueous medium. ACS Nano 2, 1252-1258 (2008).

16. Gui, R. et al. Room-temperature phosphorescence logic gates developed from nucleic acid functionalized carbon dots and graphene oxide. Nanoscale 7, 8289-8293 (2015)

17. $\mathrm{Li}, \mathrm{Z}$. et al. Direct aqueous-phase synthesis of sub-10 nm "luminous pearls" with enhanced in vivo renewable near-infrared persistent luminescence. J. Am Chem. Soc. 137, 5304-5307 (2015).

18. Abdukayum, A., Chen, J. T., Zhao, Q. \& Yan, X. P. Functional near infraredemitting $\mathrm{Cr} 3+/ \operatorname{Pr} 3+$ co-doped zinc gallogermanate persistent luminescent nanoparticles with superlong afterglow for in vivo targeted bioimaging. J. Am. Chem. Soc. 135, 14125-14133 (2013). 
19. An, Z. et al. Stabilizing triplet excited states for ultralong organic phosphorescence. Nat. Mater. 14, 685-690 (2015).

20. Bolton, O., Lee, K., Kim, H. J., Lin, K. Y. \& Kim, J. Activating efficient phosphorescence from purely organic materials by crystal design. Nat. Chem. 3, 205-210 (2011).

21. Gong, Y. et al. Achieving persistent room temperature phosphorescence and remarkable Mechanochromism from pure organic Luminogens. Adv. Mater. 27, 6195-6201 (2015).

22. $\mathrm{Yu}, \mathrm{H}$. et al. Smart utilization of carbon dots in semiconductor photocatalysis. Adv. Mater. 28, 9454-9477 (2016).

23. Lim, S. Y., Shen, W. \& Gao, Z. Carbon quantum dots and their applications. Chem. Soc. Rev. 44, 362-381 (2015).

24. Baker, S. N. \& Baker, G. A. Luminescent carbon nanodots: emergent nanolights. Angew. Chem. Int. Ed. Engl. 49, 6726-6744 (2010).

25. Xu, S., Chen, R., Zheng, C. \& Huang, W. Excited state modulation for organic afterglow: materials and applications. Adv. Mater. 28, 9920-9940 (2016).

26. Xie, Z., Wang, F. \& Liu, C. Y. Organic-inorganic hybrid functional carbon dot gel glasses. Adv. Mater. 24, 1716-1721 (2012).

27. Kwon, W. et al. Freestanding luminescent films of nitrogen-rich carbon nanodots toward large-scale phosphor-based white-light-emitting devices. Chem. Mater. 25, 1893-1899 (2013).

28. $\mathrm{Li}, \mathrm{X}$. et al. Intercrossed carbon nanorings with pure surface states as low-cost and environment-friendly phosphors for white-light-emitting diodes. Angew. Chem. Int. Ed. Engl. 54, 1759-1764 (2015).

29. Jiang, K. et al. Triple-mode emission of carbon dots: applications for advanced anti-counterfeiting. Angew. Chem. Int. Ed. Engl. 55, 7231-7235 (2016).

30. Al-Attar, H. A. \& Monkman, A. P. Room-temperature phosphorescence from films of isolated water-soluble conjugated polymers in hydrogen-bonded matrices. Adv. Funct. Mater. 22, 3824-3832 (2012).

31. Deng, Y. et al. Long lifetime pure organic phosphorescence based on water soluble carbon dots. Chem. Commun. 49, 5751-5753 (2013).

32. Tan, J., Zhang, J., Li, W., Zhang, L. \& Yue, D. Synthesis of amphiphilic carbon quantum dots with phosphorescence properties and their multifunctional applications. J. Mater. Chem. C. 4, 10146-10153 (2016).

33. Dong, X. et al. Efficient long lifetime room temperature phosphorescence of carbon dots in a potash alum matrix. J. Mater. Chem. C. 3, 2798-2801 (2015).

34. Tan, J. et al. Large-scale synthesis of $\mathrm{N}$-doped carbon quantum dots and their phosphorescence properties in a polyurethane matrix. Nanoscale 8, 4742-4747 (2016).

35. Li, Q. et al. Efficient room-temperature phosphorescence from nitrogen-doped carbon dots in composite matrices. Chem. Mater. 28, 8221-8227 (2016).

36. Garczarek, F. \& Gerwert, K. Functional waters in intraprotein proton transfer monitored by FTIR difference spectroscopy. Nature 439, 109-112 (2006).

37. Fisette, O. et al. Hydration dynamics of a peripheral membrane protein. J. Am. Chem. Soc. 138, 11526-11535 (2016).

38. Weng, L., Ziaei, S. \& Elliott, G. D. Effects of water on structure and dynamics of trehalose glasses at low water contents and its relationship to preservation outcomes. Sci. Rep. 6, 28795 (2016).

39. Gezici-Koç, Ö., Erich, S. J. F., Huinink, H. P., van der Ven, L. G. J. \& Adan, O. C. G. Bound and free water distribution in wood during water uptake and drying as measured by $1 \mathrm{D}$ magnetic resonance imaging. Cellulose 24, 535-553 (2017).

40. Wang, Y. et al. Water-mediated structuring of bone apatite. Nat. Mater. 12, 1144-1153 (2013)

41. Ball, P. Water is an active matrix of life for cell and molecular biology. Proc. Natl. Acad. Sci. USA 114, 13327-13335 (2017).

42. Yamazoe, K. et al. Enhancement of the hydrogen-bonding network of water confined in a polyelectrolyte brush. Langmuir 33, 3954-3959 (2017).

43. Nagasawa, D., Azuma, T., Noguchi, H., Uosaki, K. \& Takai, M. Role of interfacial water in protein adsorption onto polymer brushes as studied by SFG spectroscopy and QCM. J. Phys. Chem. C. 119, 17193-17201 (2015).
44. Song, Z. et al. Invisible security ink based on water-soluble graphitic carbon nitride quantum dots. Angew. Chem. Int. Ed. Engl. 55, 2773-2777 (2016).

45. Li, X., Zhang, S., Kulinich, S. A., Liu, Y. \& Zeng, H. Engineering surface states of carbon dots to achieve controllable luminescence for solid-luminescent composites and sensitive $\mathrm{Be}^{2+}$ detection. Sci. Rep. 4, 4976-4983 (2015).

46. Hirata, S. et al. Efficient persistent room temperature phosphorescence in organic amorphous materials under ambient conditions. Adv. Funct. Mater. 23, 3386-3397 (2013).

47. Zhang, J. \& Yu, S.-H. Carbon dots: large-scale synthesis, sensing and bioimaging. Mater. Today 19, 382-393 (2016).

48. Zhu, S. et al. Highly photoluminescent carbon dots for multicolor patterning sensors, and bioimaging. Angew. Chem. Int. Ed. Engl. 52, 3953-3957 (2013).

49. Ding, H., Wei, J. S. \& Xiong, H. M. Nitrogen and sulfur co-doped carbon dots with strong blue luminescence. Nanoscale 6, 13817-13823 (2014).

50. Ramon-Marquez, T., Medina-Castillo, A. L., Fernandez-Gutierrez, A. \& Fernandez-Sanchez, J. F. A novel optical biosensor for direct and selective determination of serotonin in serum by solid surface-room temperature phosphorescence. Biosens. Bioelectron. 82, 217-223 (2016).

\section{Acknowledgements}

This research was supported by the National Basic Research Program of China (973 Program, Grant No. 2011CB013004) of the Ministry of Science and Technology of China and the Major Project of Chinese State Key Laboratory of Tribology (Grant No. SKLT2014A01).

\section{Author contributions}

Q.L. and M.Z. conceived the experiments. Q.L. synthesised all materials, made all photophysical measurements and wrote the paper. M.Z., M.Y., Q.Y., Z.Z. and J.S. gave suggestions to optimise the system. All authors discussed the results and commented on the manuscript at all stages.

\section{Additional information}

Supplementary Information accompanies this paper at https://doi.org/10.1038/s41467018-03144-9.

Competing interests: The authors declare no competing financial interests.

Reprints and permission information is available online at http://npg.nature.com/ reprintsandpermissions/

Publisher's note: Springer Nature remains neutral with regard to jurisdictional claims in published maps and institutional affiliations.

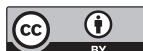

Open Access This article is licensed under a Creative Commons Attribution 4.0 International License, which permits use, sharing, adaptation, distribution and reproduction in any medium or format, as long as you give appropriate credit to the original author(s) and the source, provide a link to the Creative Commons license, and indicate if changes were made. The images or other third party material in this article are included in the article's Creative Commons license, unless indicated otherwise in a credit line to the material. If material is not included in the article's Creative Commons license and your intended use is not permitted by statutory regulation or exceeds the permitted use, you will need to obtain permission directly from the copyright holder. To view a copy of this license, visit http://creativecommons.org/ licenses/by/4.0/.

(C) The Author(s) 2018 